\title{
On the Efficiency of Sequential Auctions for Spectrum Sharing
}

\author{
Junjik Bae, Eyal Beigman, Randall Berry, Michael L. Honig, and Rakesh Vohra
}

\begin{abstract}
In previous work we have studied the use of sequential second price auctions for sharing a wireless resource, such as bandwidth or power. The resource is assumed to be managed by a spectrum broker (auctioneer), who collects bids and allocates discrete units of the resource. It is well known that a second price auction for a single indivisible good has an efficient dominant strategy equilibrium; this is no longer the case when multiple units of a homogeneous good are sold in repeated iterations. Previous work attempted to bound this inefficiency loss for two users with non-increasing marginal valuations and full information. This work was based on studying a setting in which one agent's valuation for each resource unit is strictly larger than any of the other agent's valuations and assuming a certain property of the price paid by such a dominant user in any sub-game. Using this assumption it was shown that the worst-case efficiency loss was no more than $e^{-1}$. However, here we show that this assumption is not satisfied for all non-increasing marginals with this dominance property. In spite of this, we show that it is always true for the worst-case marginals for any number of goods and so the worstcase efficiency loss for any non-increasing marginal valuations is still bounded by $e^{-1}$.
\end{abstract}

\section{INTRODUCTION}

It is becoming widely accepted that the current mechanisms for allocating wireless spectrum are not agile enough to efficiently exploit this resource. This has led to interest in a variety of different dynamic spectrum sharing approaches. One such approach is for a spectrum manager or broker to dynamically lease spectrum to secondary users for relatively short time periods. For example, in [1], [5], [6] various models with such a spectrum manager are described (see also [2]-[4] for more general discussions of secondary spectrum markets).

One natural approach for allocating spectrum to secondary users is to use an auction mechanism. Numerous auction mechanisms have been studied in the literature. Of these the Vickrey-Clarke-Groves (VCG) mechanism is a well known to yield the efficient outcome. ${ }^{1}$ However, there are pragmatic reasons to prefer alternative mechanisms over VCG. In [1] one such mechanism was considered for allocating spectrum, namely a sequential second price auction. In this mechanism,

This research was supported in part by NSF under grant CNS-0519935. Junjik Bae is with the Boston consulting Group, Seoul Korea, e-mail: baejunjik@gmail.com

Eyal Beigman is with the Olin Business School, Washington University in St. Louis (email: beigman@olin.wustl.edu).

Randall Berry and Michael Honig are with Department of Electrical Engineering and Computer Science at Northwestern University (e-mails: \{rberry, mh\}@eecs.northwestern.edu).

Rakesh Vohra is with CMS-EMS, Kellogg School of Management at Northwestern University (e-mail :r-vohra@northwestern.edu).

${ }^{1}$ For a given auction mechanism, the bidders can be viewed as playing a game, in which their actions are their bids. The auction is efficient if the equilibrium of this game maximizes the total utility of the agents. the resource is divided into discrete units and each resource unit is auctioned off sequentially according to a secondprice auction. Namely, each unit is allocated to the highest bidder, who pays the second-highest bid. In [1] such auctions were studied for allocating either the power of a particular secondary user or the total bandwidth available to all secondary users. Here, we further consider this mechanism in the context of a bandwidth auction.

Sequential auctions have been used in many applications (e.g., see [7]-[10]) since they require relatively little computation and information exchange among the agents and the broker, compared with other mechanisms. In addition, sequential auctions easily accommodate scenarios in which agents enter and leave the market at arbitrary times, and allow the broker to allocate resources incrementally. However, it is well known that sequential auctions do not always achieve an efficient allocation [9]. In work such as [9]-[17] this efficiency loss has been studied for models in which valuations are private information. Since the assumption of private information so complicates the analysis, these papers restrict attention to the case of bidders with unit demands and in some cases, to just two bidders.

In the present paper we will instead consider a model as in [1] in which bidders may have multi-unit demands but for tractability assume full information. Abstracting away from private information also allows us to focus on the strategic implications of bidding in sequential auctions. ${ }^{2}$ For such a model in [1], it was shown that for two users and an arbitrary number of resource units, the sequential second price auction always has a unique equilibrium ${ }^{3}$ allocation. Following [1], our focus is on characterizing the efficiency loss of this equilibrium for the two users and an arbitrary number of goods, when each agent has a concave utility for the spectrum resource. In [1] it was argued that this efficiency loss was at most $\frac{1}{e}$. This argument was based on studying a setting in which one user was dominant, meaning that the user's valuation for each resource unit was greater than the other user's valuation for any unit. It was assumed that in the equilibrium of the sequential auction in which such a dominant user received $l$ units, she would pay for each unit an amount equal to the other agent's marginal valuation for the $(n-l+1)$-th unit, and moreover, that the equilibrium was given by the allocation that maximized her pay-off subject to this payment property. However, as we will show, neither part of this assumption need be true for every dominant utility profile and so the proof in [1] does not apply to all decreasing

\footnotetext{
${ }^{2}$ Similar assumptions are made in [18], [19].

${ }^{3}$ See Section II for a precise definition of the equilibrium concept we use.
} 
marginal valuations. In spite of this, we will show that this property does apply for the worst-case marginal valuations and that indeed the worst-case efficiency loss is at most $\frac{1}{e}$.

\section{MOdeL}

We consider a model as in the bandwidth auction studied in [1]. There are $n$ resource units to be allocated among $k=2$ agents via a sequential second-price auction. In this auction, the units are allocated sequentially in $n$ rounds. In round $m \leq n$, each agent submits a bid for the $m$ th unit. The auctioneer allocates this unit to the agent with the largest bid and charges that agent the second largest bid.

This mechanism can be viewed as an extensive form game with a balanced binary game tree. Each decision node in the game tree designates a state of the world, where a certain quantity of goods (resource units) are allocated to the two agents. Let $\mathbf{s}=\left(s_{1}, s_{2}\right)$ denote such an allocation. Since the goods are homogeneous, the decision nodes with the same allocation can be unified and the game tree can be replaced with a directed graph $G=(V, E)$, where $V=\left\{\mathbf{s} \in[1, \ldots, n]^{2} \mid s_{1}+s_{2} \leq n\right\}$ (see Fig. 1). A node $\mathbf{s} \in V$ represents the outcome of the $\left(s_{1}+s_{2}\right)$-th round, in which agent $i$ has been allocated $s_{i}$. For $s_{1}+s_{2}<n$, each node $\mathbf{s}$ has directed edges to the two children $\left(s_{1}+1, s_{2}\right)$ (corresponding to agent 1 winning the current round) and $\left(s_{1}, s_{2}+1\right)$ (corresponding to agent 2 winning). The auction begins at the root node $(0,0)$.

Let $u_{j}^{i}$ denote the marginal valuation of agent $i$ for the $j$ th unit. We assume that both agents have non-increasing marginal valuations so that $u_{1}^{i} \geq u_{2}^{i} \geq \cdots \geq u_{n}^{i}$. Agent $i$ 's total valuation for receiving $s_{i}$ units is therefore $\sum_{j=1}^{s_{i}} u_{j}^{i}$. Let $H$ designate the set of observable bidding histories. A strategy $\sigma_{i}: V \times H \rightarrow \mathbb{R}^{+}$is a function mapping states of the allocation and observable histories to bids. The strategy set of an agent is the set of all such functions. The outcome path of a strategy profile $\left\{\sigma_{1}, \sigma_{2}\right\}$ is a directed path $\delta=\left\{\mathbf{s}^{1}, \ldots, \mathbf{s}^{n}\right\}$ in $G$ such that if $s_{i}^{t+1}=s_{i}^{t}+1$ and $s_{j}^{t+1}=s_{j}^{t}$ for $j \neq i$ then $\sigma_{i}\left(\mathbf{s}^{t}, \Gamma_{t}\right) \geq \sigma_{j}\left(\mathbf{s}^{t}, \Gamma_{t}\right)$, for all $j \neq i$, where $\Gamma_{t}$ is the bidding history of the first $t$ units. ${ }^{4}$ The total payment of agent $i$ along the path $\delta$ is $P_{i}(\delta)=\sum_{t=1}^{n} p_{i}\left(\mathbf{s}^{t}\right)$, where for each $\mathbf{s}^{t} \in \delta, p_{i}\left(\mathbf{s}^{t}\right)=\sigma_{j}\left(\mathbf{s}^{t}, \Gamma_{t}\right), j \neq i$, if $s_{i}^{t+1}=s_{i}^{t}+1$ and $p_{i}\left(\mathbf{s}^{t}\right)=0$, otherwise.

Here we focus on sophisticated bidding strategies in which an agent maximizes her payoff over final outcomes. The ability to make inferences on the final outcome requires that the agent be sufficiently informed about the preferences and strategies of the other agent. Here, we assume full information, i.e., each agent knows the number of units being sold, bidding histories, and the valuations of the other agent. A similar analysis could be made for the case where the agent is Bayesian and knows the distribution of the other agent's marginal values.

\footnotetext{
${ }^{4}$ In the case of ties, any tie-breaking rule that allocates the good to one of the agents can be used.
}

\section{ANALYSIS}

First we review the analysis in [1] regarding the outcome of the preceding auction for two agents with sophisticated bidding and full information. Since all agents know when the last unit is being sold, regardless of the bidding history, the last round of the auction is a standard second-price auction for the $n$-th good. (The values for this good will, of course, depend on the outcomes of the previous rounds). Hence it is a weakly dominant strategy for the agents to bid their marginal values on the last round. ${ }^{5}$ Since those values are common knowledge, all agents know beforehand the allocation and payments in the last round. Thus, we can think of the penultimate round as an auction over the right to participate in one of two auctions in the last round. Since the payoffs of each one of those auctions is common knowledge, we can think of the penultimate round as a second-price auction with valuation equal to payoff difference between those two auctions. It is therefore a weakly dominant strategy in the penultimate round to bid the payoff difference associated with the outcomes of the two auctions in the last round.

We can proceed in this way inductively until we reach the root. This shows that sophisticated bidding is the only strategy that survives iterative elimination of weakly dominated strategies. ${ }^{6}$ This does not rule out other equilibria and in fact there may exist other Nash equilibria with higher payoffs for both agents (if, for example, they conspire against the seller). However, those equilibria must rely on unreliable threats and commitments. We eliminate those equilibria from consideration by focusing on subgame perfect equilibria that survive the iterative elimination of weakly dominated strategies. $^{7}$ This discussion is summarized in the following theorem.

Theorem 1: With two fully informed agents, the sophisticated bidding equilibrium is the only subgame perfect equilibria that survives iterative elimination of weakly dominated strategies.

We define the equilibrium path to be the outcome path when both agents use a sophisticated bidding strategy, and the sequential allocation to be the allocation at the terminal node of the equilibrium path. From the previous discussion if all agents apply a sophisticated bidding strategy, then all equilibria have the same equilibrium path, and the same (unique) sequential allocation.

Example 1: Consider a sequential auction with $n=2$ units. Figure 1 (c) shows the directed graph $G$ with each node labeled by the allocation $\left(s_{1}, s_{2}\right)$. Assume that $u_{1}^{1}=u_{2}^{1}=5$, $u_{1}^{2}=4$ and $u_{2}^{2}=1$. Since agent 1 values each unit more than agent 2 values any unit, the efficient allocation is to give both units to agent 1 .

Now let us examine sophisticated bidding for this example.

\footnotetext{
${ }^{5}$ A strategy is weakly dominant for an agent if no other strategy gives that agent a larger pay-off, for any choice of strategies for the other agents.

${ }^{6}$ In other words all strategies which are weakly dominated are removed from consideration [20].

${ }^{7} \mathrm{~A}$ subgame perfect equilibrium is a refinement of the concept of Nash equilibrium with the restriction that agents cannot make non-credible threats [20].
} 

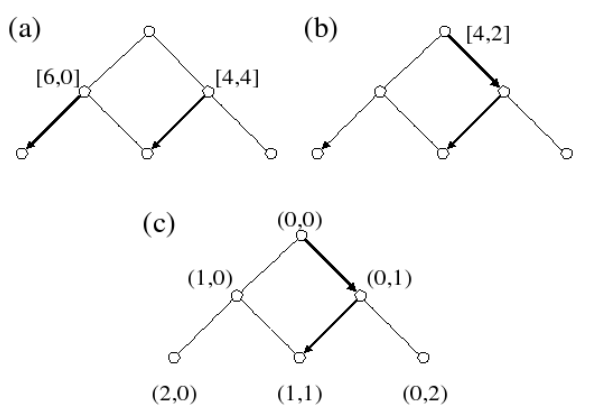

Fig. 1. Example of the sequential auction with $k=2$ agents and $n=2$ resource units. (a) and (b) show the valuations of each node and (c) shows the equilibrium path.

Assume that the game reaches node $v=(1,0)$, so that the agents bid for the one remaining unit, given that the first unit has gone to agent 1. (See Fig. 1 (a).) In this stage it is weakly dominate for the agents to bid their valuations, i.e., agent 1 bids $u_{2}^{1}=5$ and agent 2 bids $u_{1}^{2}=4$. The auctioneer then allocates the unit to agent 1 and charges her a price of 4 . Hence the value of node $v=(1,0)$ to agent 1 is $u_{1}^{1}+\left(u_{2}^{1}-u_{1}^{2}\right)=6$, where $u_{1}^{1}$ is the value from winning the first unit and $u_{2}^{1}-u_{1}^{2}$ is the surplus for winning the second unit. The value of $v=(1,0)$ to agent 2 is 0 . Similarly, the value of $v=(0,1)$ is 4 to either agent. Given these values, the agents can optimize their bids for the first unit. In particular, agent 1 bids her marginal valuation, which is $6-4=2$, and agent 2 bids $4-0=4$. It follows that agent 2 wins the first unit and pays 2. Therefore the equilibrium path is $\delta=\{(0,0),(0,1),(1,1)\}$, i.e., each user receives one unit. Note that $\delta$ does not terminate in an efficient allocation. In what follows, we characterize the efficiency loss of this equilibrium.

\section{A. Worst-case efficiency loss}

Given $n$ resource units and two agents, let $(l, n-l)$ denote the efficient allocation, and $\left(l^{\prime}, n-l^{\prime}\right)$ denote the sequential allocation. The worst-case efficiency is defined by

$$
\eta(n)=\min _{\left\{u_{i}^{1}\right\},\left\{u_{i}^{2}\right\} \in \mathcal{U}} \frac{\sum_{i=1}^{l^{\prime}} u_{i}^{1}+\sum_{i=1}^{n-l^{\prime}} u_{i}^{2}}{\sum_{i=1}^{l} u_{i}^{1}+\sum_{i=1}^{n-l} u_{i}^{2}} .
$$

That is, the worst-case is with respect to the marginal values in some admissible set $\mathcal{U}{ }^{8}$ We refer to $1-\eta(n)$ as the worstcase efficiency loss. Our main result given in the following theorem is to characterize this worst-case loss when $\mathcal{U}$ is the set of all non-increasing marginal valuations.

Theorem 2: In a two-agent sequential second-price auction with non-increasing marginal values $\eta(n) \geq 1-e^{-1}$.

In other words, the worst case efficiency loss is bounded by $e^{-1}$. Moreover, it can be shown that $\eta(n)$ decreases with $n$, and the bound $1-e^{-1}$ is asymptotically tight as $n \rightarrow \infty$.

\footnotetext{
${ }^{8}$ The quantity $1 / \eta(n)$ is similar to the "price of anarchy" as in [18], [19], except that we are using a stricter equilibria concept here.
}

1) Dominant Utility Profiles: An attempt to prove Theorem 2 was given in [1] that was based on studying the family of utility functions defined next.

Definition 1: Agent 1's utilities are dominant if $u_{1}^{1} \geq$ $\ldots \geq u_{n}^{1} \geq u_{1}^{2} \geq \ldots \geq u_{n}^{2}$. We will also refer to this as a dominant utility profile. Agent 1's utilities are flat dominant if $u_{1}^{1}=\ldots=u_{n}^{1} \geq u_{1}^{2} \geq \ldots \geq u_{n}^{2}$.

The efficient allocation for a dominant utility profile is to assign all units to agent 1 . In the sequential allocation, however, agent 2 may receive up to $n-1$ units (e.g. this is the case in Example 1).

In [1], it was (incorrectly) argued that for agents with a dominant utility profile the following properties are always true:

Property D1: In the sequential allocation $(s, t)$ agent 1 pays $u_{n-s+1}^{2}$ for each unit she receives.

Property D2: The sequential allocation $(s, t)$ satisfies

$$
s=\arg \max _{\tilde{s} \leq n} \sum_{i=1}^{\tilde{s}}\left(u_{i}^{1}-u_{n-\tilde{s}+1}^{2}\right) .
$$

Note that with a dominant utility profile, agent 1 will always win at least the last unit and thus in Property D1, $s \geq 1$. Property D2 states that in the sequential allocation, agent 1 receives the maximum pay-off she could over all allocations if her pay-offs were subject to satisfying Property D1. Hence, we refer to an allocation that satisfies (1) as the dominant user optimal allocation.

Referring to Example 1, note that the agents have a dominant utility profile and in the sequential allocation, agent 1 's payment satisfies the Property D1. Moreover, it can easily be seen that this is also the dominant user optimal allocation (i.e., it satisfies D2).

In [1], assuming both of these properties the worst-case efficiency for an auction with $n$ goods and a flat dominant profile was characterized. Furthermore it was argued that the worst-case efficiency over all (non-increasing) valuations for a given number of goods is always achieved by a flat dominant profile. Combining all of these observations, it was argued that $\eta(n) \rightarrow 1-e^{-1}$ as the number of goods increases. Furthermore, in [1] flat dominant utility profiles which asymptotically achieve this bound were constructed. These profiles all satisfy both Properties D1 and D2. However, as the next example illustrates, Properties D1 and D2 need not hold for all flat dominant profiles.

Example 2: Consider an auction with $n=3$ goods and $k=2$ agents. Suppose that the agents have the following flat dominant utility profiles: $u_{1}^{1}=u_{2}^{1}=u_{3}^{1}=1, u_{1}^{2}=u_{2}^{2}=$ $2 / 3-\epsilon$ and $u_{3}^{2}=0$. In other words, agent 1 values each unit at 1 while agent 2 only values the first two units at $2 / 3-\epsilon$ each, where $\epsilon$ is some small number. It can be shown that for this example the sequential allocation is to give 1 unit to agent 1 and two units to agent 2 . However, the dominant user optimal allocation is for agent 1 to receive all three units. The problem here is that at the first stage, if agent 2 wins, she will also win at the next stage, while if she loses she will not win anything. Hence her bid at the first stage is 
$1-3 \epsilon$, which for small enough $\epsilon$ is larger than her valuation for the first good $(2 / 3-\epsilon)$. Thus to win, user 1 would have to bid at a larger value than that predicted by Property D1.

In this example, user 1's payment for the good she receives in the sequential allocation does satisfy Property D1, however if we instead changed user 2's utility profile to be $u_{1}^{2}=u_{2}^{2}=1 / 2+\epsilon$ and $u_{3}^{2}=0$, then the sequential allocation becomes $(3,1)$ but user 1 will pay $1 / 2+3 \epsilon$ for the first unit she receives, which does not satisfy Property D2.

Because of this example, it follows that the proof in [1] does not apply to all flat dominant utility profiles and so the worst-case efficiency bound given there may also not apply.

2) A Second Try: We denote the worst-case efficiency by $\eta^{\prime}(n)$ in an auction with $n$ goods when the agents are constrained to having a flat dominant utility profile. As in [1] we will again first bound the efficiency loss assuming that the utility functions are in this class and then argue that this class also gives the worst-case efficiency over all possible valuations.

To begin we first explicitly derive $\eta^{\prime}(n)$ for $n=2$ goods in the following example.

Example 3: As in Example 1 consider an auction with $n=2$ goods and $k=2$ agents with a flat dominant utility profile. Without loss of generality, we assume $u_{1}^{1}=u_{2}^{1}=1$ for agent 1 and $u_{1}^{2}=b_{1}, u_{2}^{2}=b_{2}$ for agent 2 . Note that $1 \geq$ $b_{1} \geq b_{2}$ with a flat dominant utility profile. Using backward induction as in Example 1, the value of node $v=(1,0)$ is $\left[2-b_{1}, 0\right]$. Similarly, the value of node $v=(0,1)$ is $\left[1-b_{2}, b_{1}\right]$. Given these values, agent 1 's bid for the first unit is $\left(2-b_{1}\right)-\left(1-b_{2}\right)=1-b_{1}+b_{2}$ and agent 2 's bid for the first unit is $b_{1}$. If $1-b_{1}+b_{2}>b_{1}$, then agent 1 wins the first unit and pays $b_{1}$. In this case, the sequential allocation is $(2,0)$ and there is no efficiency loss. On the other hand, if $1-b_{1}+b_{2}<b_{1}$, the sequential auction reaches the terminal node $(1,1)$ and the efficiency of the auction becomes $\frac{1+b_{1}}{1+1}$. From the set of constraints

$$
\left\{\begin{array}{l}
1>b_{1}>b_{2} \\
1-b_{1}+b_{2}<b_{1}
\end{array}\right.
$$

to have the equilibrium path $\delta=\{(0,0),(0,1),(1,1)\}$, it can be shown that the worst-case efficiency in this case occurs when $b_{1}=1 / 2+\epsilon$ and $b_{2}=0$, and so $\eta^{\prime}(2)=$ $\lim _{\epsilon \rightarrow 0} \frac{1+b_{1}}{2}=3 / 4$. Interestingly, with the worst-case utility profile Properties D1 and D2 hold, and indeed $3 / 4$ is the same as the worst-case efficiency given in [1] for $n=2$ goods.

Directly generalizing the approach in Example 3 to an arbitrary number of goods is complex due to the increase in the number of constraints that must be accounted for. To simplify this we first further restrict the class of flat dominant utility profiles to a class that supports subgame perfect equilibrium paths along which agent 2 consumes the first $n-j$ units and agent 1 consumes the remaining $j$ units in that order. Later, we show that these profiles give the worst-case efficiency among all flat dominant utility profile.
Definition 2: A node in an equilibrium path is a "kink" if immediately prior to that node (on the equilibrium path) one agent wins and immediately following the other agent wins.

Definition 3: A profile of marginal utilities is said to have the subgame kink property if (1) each path that corresponds to a subgame perfect equilibrium of the entire game has at most one kink, and (2) each path that corresponds to an equilibrium path for a subgame starting at any node not on a path in (1) has zero kinks.

Definition 4: The subgame perfect equilibrium is called a subgame kink when the profile of marginal utilities has the subgame kink property.

Note that if a flat dominant utility has the subgame kink property is must be that in equilibrium agent 2 consumes the first $n-j$ units (for some $j<n$ ) and agent 1 consumes the remaining $j$ units. Additionally, the resulting allocation will satisfy Property D1. The following lemma shows that for any $j \geq 1$, we can find a flat dominant utility profile with the subgame kink property that results in that allocation.

Lemma 3: For any allocation $(j, n-j)$ in a $n$-unit auction with $j \geq 1$, there is a flat dominant utility profile with the subgame kink property for which $(j, n-j)$ is the sequential allocation.

Proof: The following profile of marginal utilities has the subgame kink property with the sequential allocation $(j, n-j): u_{1}^{1}=\cdots=u_{n}^{1}=1$ for agent 1 and

$$
\begin{cases}u_{i}^{2}=1-\frac{j}{n-i+1}+\epsilon_{i}, & i=1, \cdots, n-j, \\ u_{i}^{2}=0, & i=n-j+1, \cdots, n,\end{cases}
$$

for agent 2. Note that $\epsilon_{i} \rightarrow 0^{+}$for all $i$. Backward induction verifies that this results in a subgame kink.

Note that when $\epsilon_{i} \rightarrow 0$ with these marginal utilities, all subgame kinks with the sequential allocations $(i, n-i)$ for $i=j, \cdots, n$ can be supported in equilibrium with the values of the root node $[j, 0]$. In addition, the following relations among the marginal utilities of agent 2 hold

$$
\begin{aligned}
n-n \cdot u_{1}^{2} & =(n-1)-(n-1) \cdot u_{2}^{2} \\
& \vdots \\
& =(j+1)-(j+1) \cdot u_{n-j}^{2} \\
& =j .
\end{aligned}
$$

Essentially this shows that Property D2 holds for these marginals.

Lemma 4: For $n=2$, the worst-case efficiency among the flat dominant utility profiles is achieved by one with the subgame kink property.

Proof: We have shown in Example 3 that the worstcase efficiency with $n=2$ is given by a flat dominant utility profile with $u_{1}^{1}=u_{2}^{1}=1, u_{1}^{2}=1 / 2$ and $u_{2}^{2}=0$ and this profile induces a subgame kink.

For given $b \leq 1$, let $h^{\prime \prime}(n, b)$ be the worst-case efficiency in a $n$-unit auction among all flat dominant utility profiles with the subgame kink property and for which $b_{1} \leq b$ (i.e., agent 2's marginal utility for the first unit is no greater than $b$ ). For example, for $n=2$, if $b<\frac{1}{2}$, then $h^{\prime \prime}(2, b)=1$, 
while for $b \geq \frac{1}{2}, h^{\prime \prime}(2, b)=3 / 4$. Note that the first case is achieved when agent 2 has marginal utilities $b_{1}=b_{2}=0$, which correspond to the profile in Lemma 3 for $j=2$; the second case is achieved by the marginal utilities $b_{1}=\frac{1}{2}$ and $b_{2}=0$ (Lemma 3 with $j=1$ ).

Lemma 5: For all $n, h^{\prime \prime}(n, b)$ is achieved by one of the profiles in Lemma 3.

Proof: This is proved by induction on $n$. We have already shown that this is true for $n=2$. Assume it is true for $n-1$ and consider the worst-case efficiency of a subgame kink for $n$ units. If the first unit goes to agent 1 , we are done since the only subgame kink is then for agent 1 to win everything, which corresponds to the marginal utilities in Lemma 3 for $j=n$.

If the first unit goes to agent 2 with a marginal utility of $b_{1}<b$, then the worst-case efficiency on the subtree from the node $(0,1)$ must be given by $h^{\prime \prime}\left(n-1, b_{1}\right)$. By the induction hypothesis this is achieved on the subtree by a set of marginal utilities $b_{2}, b_{3}, \ldots, b_{n}$ given by Lemma 3 for some $j$. Furthermore, the worst-case efficiency is $\left(b_{1}+\right.$ $\left.(n-1) \cdot h^{\prime \prime}\left(n-1, b_{1}\right)\right) / n$.

If $b_{2}, \ldots, b_{n}$ satisfy Lemma 3 for some $j$ and $n-1$ units, then, in particular, $b_{2}=1-\frac{j}{n-1}$. At the root node of the $n$-unit auction, for agent 2 to win the first unit in a subgame kink, it must be that $1-(n-1) \cdot b_{1}+(n-1) \cdot b_{2}<b_{2}$, or

$$
n \cdot\left(1-b_{1}\right)<(n-1) \cdot\left(1-b_{2}\right) \text {. }
$$

By combining this inequality with $b_{2}=1-\frac{j}{n-1}$, it can be seen that in the worst-case efficiency it should be that $b_{1}=1-\frac{j}{n}$, which corresponds to one of the profiles in Lemma 3.

Lemma 6: For all $n$ and $b \leq 1$,

$$
\frac{1+(n-1) h^{\prime \prime}(n-1, b)}{n} \geq h^{\prime \prime}(n, b) \text {. }
$$

Proof: Suppose $h^{\prime \prime}(n-1, b)$ is achieved when agent 1 receives $j$ units. From Lemma 5, it follows that this is achieved by one of the profiles in Lemma 3 and so $1-\frac{j}{n-1}<$ $b$.

Consider the marginal utilities from Lemma 3 for $n$ units where agent 1 receives $j+1$ units and note that

$$
1-\frac{j+1}{n}<1-\frac{j}{n-1}
$$

so that this marginal will satisfy $b_{1}<b$. Let $\tilde{h}(n, b)$ denote the efficiency achieved with these marginal utilities. It can be seen that

$$
\begin{aligned}
n-n \cdot \tilde{h}(n, b) & =\sum_{i=1}^{n-(j+1)} \frac{j+1}{n-i+1} \\
& \geq \sum_{i=1}^{(n-1)-j} \frac{j}{(n-1)-i+1} \\
& =(n-1)-(n-1) \cdot h^{\prime \prime}(n-1, b),
\end{aligned}
$$

or

$$
\frac{1+(n-1) \cdot h^{\prime \prime}}{n} \geq \tilde{h}(n, b) \geq h^{\prime \prime}(n, b)
$$

The bound then follows from noting that $h^{\prime \prime}(n, b)$ must be no greater than $\tilde{h}(n, b)$.

Corollary 7: Amongst all the flat dominant profiles with the subgame kink property, the worst-case efficiency is $h^{\prime \prime}(n, 1)$, where $h^{\prime \prime}(n, 1)$ can be obtained explicitly from Lemma 3 and is given by

$$
\eta^{\prime \prime}(n) \equiv h^{\prime \prime}(n, 1)=\min _{j \in[1, \cdots, n]}\left\{1-\frac{j}{n} \sum_{i=j}^{n-1} \frac{1}{i+1}\right\} \text {. }
$$

Now we are ready to prove the worst-case efficiency of the sequential auction with a flat dominant utility profile. For given $b \leq 1$, let $h^{\prime}(n, b)$ be the worst-case efficiency in a $n$-unit auction among all flat dominant profiles for which $b_{1} \leq b$. Note that $\eta^{\prime}(n)=h^{\prime}(n, 1)$.

Theorem 8: For all $n, h^{\prime}(n, b)$ is achieved by one of the profiles in Lemma 3, i.e., $h^{\prime}(n, b)=h^{\prime \prime}(n, b)$. Especially when $b=1$, then $\eta^{\prime}(n)=\eta^{\prime \prime}(n)$.

Proof: We prove this by induction on $n$. From Lemma 4, it is true for $n=2$. Suppose it is true for $n-1$ and consider a $n$-unit auction with a given constant $b$. Let $b_{1} \leq b$ be agent 2 's marginal in the worst-case. If the first unit goes to agent 1 , then by induction the worst-case from $(1,0)$ is a subgame kink with an efficiency at least $h^{\prime \prime}\left(n-1, b_{1}\right)$. Suppose that agent 2 wins the second unit, then the worstcase efficiency would be

$$
\frac{1+(n-1) \cdot h^{\prime \prime}\left(n-1, b_{1}\right)}{1+n-1} \geq h^{\prime \prime}\left(n, b_{1}\right),
$$

from Lemma 6. If agent 1 wins the second unit, then by induction, the subgame starting at $(1,0)$ has no kinks, and so $h^{\prime}(n, b)=1$, which is trivially assumed by the marginal utilities in Lemma 3 for $j=n$.

On the other hand, if the first unit goes to agent 2, by induction we have a subgame kink from the node $(0,1)$ for any marginal utility of $b_{1}$ due to Lemma 5 . Now we have to show that the subtree starting from the node $(1,0)$ is a line with no kinks for the worst-case efficiency with $n$ units. Given agent 2 wins the first unit in the worst-case, the marginals for $b_{2}, \ldots, b_{n}$ must satisfy Lemma 3 and we have $b_{2}=1-\frac{j}{n-1}$ for some $j$. Suppose that the subtree starting from the node $(1,0)$ in the worst-case is not a line. Then it must be that $b_{1}<1-\frac{j}{n}$. Otherwise, we could lower $b_{1}$ to this value and get the subgame kink in Lemma 3 with a lower efficiency. Furthermore, if this subtree is not a line, it must be that for some $m<n$, at node $(n-m, 0)$, $m \cdot\left(1-b_{1}\right)<(m-1) \cdot\left(1-b_{2}\right)$. However, this can only be true if $b_{1}>1-\frac{j}{n}$, which contradicts the above. Therefore, the subtree from the node $(1,0)$ is a line with no kinks for the worst-case efficiency.

From Corollary 7 and Theorem 8, the worst-case efficiency of the sequential auction with the flat dominant utility profile $\eta^{\prime}(n)$ is given by (7), which converges to $1-e^{-1}$ as $n \rightarrow \infty$. To complete the proof of Theorem 2, we use the following lemma.

Lemma 9: For any number of goods $n$, the worst-case efficiency is achieved with a flat dominant utility profile. 


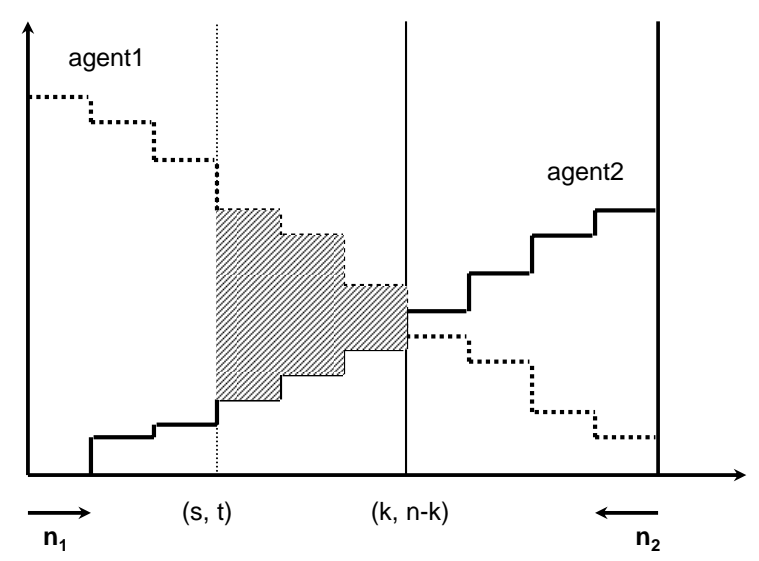

Fig. 2. Marginal values of two agents. $n_{1}\left(n_{2}\right)$ is the number of units that agent 1 (2) obtains along the sequential auction. $(l, n-l)$ is the optimal allocation and $(s, t)$ is the sequential allocation. The shadowed region shows the efficiency loss.

Proof: Suppose that $u_{1}^{1} \geq \ldots \geq u_{n}^{1}$ and $u_{1}^{2} \geq \ldots \geq$ $u_{n}^{2}$, and let $(l, n-l)$ denote the efficient allocation. After auctioning $m(\leq n)$ units, the sequential game reaches a decision node where either agent 1 or agent 2 obtains her efficient allocation ( $l$ for agent 1 or $n-l$ for agent 2). For that agent the marginal values of the remaining units must be smaller than that for the other agent. (See Figure 2.) Up to this decision node, there is no loss in efficiency. Any efficiency loss in the final allocation procures in the subgame tree rooted at this decision node. Therefore, the efficiency loss of the full game tree cannot be larger than the efficiency loss of this subgame tree. Since the utility profile associated with the subgame tree is dominant, the worst-case efficiency must always correspond to a dominant utility profile.

We now show that changing a dominant utility profile to a flat dominant utility profile can only decrease efficiency. We assume that the sequential allocation is $(s, t)$ with the following flat dominant utility profile: $u_{1}^{1}=\cdots=u_{n}^{1} \geq$ $u_{1}^{2} \geq \cdots u_{n}^{2}$. Now if we change the marginal utilities of agent 1 to $\bar{u}_{1}^{1} \geq \cdots \geq \bar{u}_{n}^{1}=u_{n}^{1}$, then the sequential allocation should be $(\bar{s}, \bar{t})$, where $\bar{s} \geq s$. This can be verified easily with the tree of the extensive form game. At every node, agent 1's bidding becomes larger with the marginal utilities $\bar{u}_{i}^{1}$, either agent 1 wins a unit otherwise agent 2 would win or agent 2 has to pay more, which implies $\bar{s} \geq s$. Due to $\bar{u}_{i}^{1} \geq u_{i}^{1}$ and $\bar{s} \geq s$, therefore, the efficiency of the sequential auction can only be lowered by changing the dominant utility profile to a flat dominant utility profile.

3) Worst-case utility profiles: Table I shows some examples of the marginal utilities that give the lowest efficiency $\eta(n)$, which is also shown. As can be seen, $\eta(n)$ is decreasing with $n$. As $n \rightarrow \infty$, these quantities approach the bound from Theorem 2.

We note that these are exactly the same as the constructive examples given in [1]. Also note that in these examples Properties D1 and D2 hold.

\begin{tabular}{|c|c|c|c|}
\hline $\mathrm{n}$ & Marginal utilities & $j^{*}$ & $\eta(n)$ \\
\hline 2 & 1,$1 ; 1 / 2+\varepsilon_{1}, 0$ & 1 & $3 / 4$ \\
\hline 3 & $1,1,1 ; 2 / 3+\varepsilon_{1}, 1 / 2+\varepsilon_{2}, 0$ & 1 & $13 / 18$ \\
\hline 4 & $1,1,1,1 ; 1 / 2+\varepsilon_{1} ; 1 / 3+\varepsilon_{2}, 0,0$ & 2 & $17 / 24$ \\
\hline$\vdots$ & $\vdots$ & $\vdots$ & $\vdots$ \\
\hline$\infty$ & & & $1-\frac{1}{e}$ \\
\hline
\end{tabular}

TABLE I

MARGINAL UTILITIES AND CORRESPONDING WORST-CASE EFFICIENCY ACHIEVED BY TWO USER THE SEQUENTIAL AUCTION FOR GIVEN $n$.

\section{CONCLUSiOnS}

We have considered a sequential second price auction for allocating $n$ units of bandwidth among non-cooperative wireless devices. This mechanism is relatively simple and requires little information exchange among users, which may make it attractive for dynamic bandwidth allocation among secondary users who wish to share spectrum with the primary user (spectrum owner or licensee). Our main result is to characterize the worst-case efficiency of the subgame perfect equilibrium for two users with full knowledge of bidding histories and user utilities. Earlier work attempted to characterize this but did so under an incorrect assumption about the equilibria in auctions in which one user was dominate. Here we show that though this assumption was wrong, the conclusions were correct and that for decreasing marginal utilities, the worst-case efficiency decreases with $n$ and converges to $1-e^{-1}$.

Although the worst-case efficiency loss due to sophisticated bidding can be significant, simulation results reported in [1] with randomly placed users show that with the rate utility function, the sequential auction typically gives the efficient allocation. Furthermore, when the equilibrium is inefficient, the efficiency loss is typically less than the worstcase efficiency loss. This is due to the rate utility function, which places constraints on the ratios of marginal utilities for the successive units being auctioned.

\section{REFERENCES}

[1] J. Bae, E. Beigman, R. Berry, M. Honig, and R. Vohra, "Sequential Bandwidth and Power Auctions for Distributed Spectrum Sharing," IEEE Journal on Selected Areas in Communication, vol. 26, no. 7, pp. 1193-1203, Sept. 2008.

[2] FCC, "The development of secondary markets - report and order and further notice of proposed rule making," Federal Communications Commission Report 03-113, 2003.

[3] - "Second report and order: Promoting efficient use of spectrum through elimination of barriers to the development of secondary markets,' Federal Communications Commission Report 04-167, Sept. 2004.

[4] J. M. Peha and S. Panichpapiboon, "Real-time secondary markets for spectrum," Telecommunications Policy, vol. 28, pp. 603-618, 2004.

[5] C. Raman, R. D. Yates, and N. B. Mandayam, "Scheduling variable rate links via a spectrum server," in New Frontiers in Dynamic Spectrum Access Networks (DySPAN), 2005, pp. 110-118.

[6] J. Huang, R. Berry, and M. L. Honig, "Auction-based spectrum sharing," ACM/Springer Mobile Networks and Applications Journal (MONET), vol. 11, pp. 405-418, 2006.

[7] C. Boutilier, M. Goldszmidts, and B. Sabata, "Sequential auctions for the allocation of resources with complementarities," in Proceedings of 16th International Joint Conference on Artificial Intelligence (IJCAI99), 1999, pp. 527-534. 
[8] J. T. yi Wang, "The ebay market as sequential second price auctions: Theory and experiments," November 2006, unpulbished manuscript.

[9] P. R. Milgrom and R. J. Weber, "A theory of auctions and competitive bidding," Econometrica, vol. 50, no. 5, pp. 1089-1122, September 1982.

[10] R. J. Weber, "Multiple-object auctions," 1983, discussion Papers 496, Northwestern University, Center for Mathematical Studies in Economics and Management Science.

[11] A. Ortego-Reichert, Models for Competitive Bidding under Uncertainty. Stanford University, 1968.

[12] D. B. Hausch, "Multi-object auctions: Sequential vs. simultaneous sales," Management Science, vol. 32, no. 12, pp. 1599-1610, December 1986.

[13] S. Bikhchandani, "Reputation in repeated second-price auctions," Journal of Economic Theory, vol. 46, no. 1, pp. 97-119, October 1988.

[14] S. Hon-Snir, D. Monderer, and A. Sela, "A learning approach to auctions," Journal of Economic Theory, vol. 82, no. 1, pp. 65-88, September 1998.

[15] M. R. Preston and V. Daniel, "The declining price anomaly," Journal of Economic Theory, vol. 60, no. 1, pp. 191-212, 1993.

[16] M. Kwiek, "Reputation and cooperation in the repeated second-price auctions," discussion Paper Series In Economics And Econometrics from University of Southampton, Economics Division, School of Social Sciences.

[17] J. Horner and J. Jamison, "Private information in repeated auctions," The Review of Economic Studies, 2008.

[18] R. Johari and J. N. Tsitsiklis, "Efficiency loss in a network resource allocation game," Mathematics of Operations Research, vol. 29, no. 3, pp. 407-435, August 2004.

[19] T. Roughgarden and E. Tardos, "How bad is selfish routing?" Journal of the ACM, vol. 49, no. 2, pp. 236-259, March 2002.

[20] M. Osborne and A. Rubinstein, A course in Game Theory. MIT Press, 1994. 